\title{
Scholte Wave Attenuation Estimates from Two Diverse Test Sites
}

\author{
Michael K. Broadhead, Hassan B. Ali, L. Dale Bibee \\ Naval Research Laboratory, Stennis Space Center, MS 39529-5004
}

\begin{abstract}
Scholte wave arrivals at two diverse test sites were analyzed with the spectral ratio method to obtain estimates of the shear attenuation properties of deep sea bottom sediments. The quantities obtained are interpreted as depth averaged estimates of the quality factor $Q$. The Scholte waves were generated by bottom located explosive charges and recorded on ocean bottom seismometers. The source-to-receiver ranges were approximately 0.6 to $2.0 \mathrm{~km}$, and the bandwidth of the Scholte waves used in the analysis was approximately 0.3 to $6.0 \mathrm{~Hz}$. The results obtained are internally consistent and fall within the range of values previously published in the literature. The results from one site are compared to $Q$ estimates reported by another investigator for an experiment site in the same area.
\end{abstract}

\section{INTRODUCTION}

The characteristics of Scholte waves are strongly affected by the shear properties of the first fifty meters of the ocean bottom. Consequently, they are a valuable tool to probe surficial sediments. For a review of efforts in this area, refer to [1]-[4]. In this paper we investigate the attenuation properties of Scholte wave measurements to obtain estimates of depth-averaged shear $Q$ for two deep water data test sites off the west coast of the U.S. Site B consists of a thin layer of hemipelagic muds overlying sand, while site $C$ is characterized by a thick section of deep sea clays. The data were collected using a remote technique in which explosive charges placed on the sea floor were used to generate Scholte waves. These were then recorded on ocean bottom seismometers (OBS) [5]-[7].

We used the spectral ratio method [8]-[10] to obtain estimates of the dimensionless parameter $Q$, which is inversely proportional to attenuation. The range of $Q$ estimates we obtained falls within generally reported ranges in the literature [3], and are self-consistent. There was not enough discrimination in the results to conclude a difference in attenuation between the two test sites. Site $\mathrm{C}$ data was collected within a few miles of the area for which Sauter et al. [9], [10] reported $Q$ estimates. We compare our results with theirs later in the paper.

\section{TEST SITE CHARACTERIZATION}

Site B was located at the base of the Oregon continental slope on the distal edge of the Astoria deep-sea fan and consists of a thin layer $(\approx 3 \mathrm{~m})$ of hemipelagic muds overlying sand. The water depth there is approximately $2600 \mathrm{~m}$. Refer to Fig. 1. Site $\mathrm{C}$ is in a sediment pond at the base of the slope defining the western edge of the Southern California borderland terrain and is characterized by a thick section of deep sea clays. Water depth at this site is approximately $3800 \mathrm{~m}$. In both cases, the current sedimentary regime is thought to be low-energy.

\section{EXPERIMENTAL PROCEDURE AND RESULTS}

The experimental procedure is described in detail in [5]-[7]. In Fig. 2 (a)-(c), we show three vertical component time series recorded on OBS 38 at site $C$ for ranges $620 \mathrm{~m}$, $1190 \mathrm{~m}$, and $2010 \mathrm{~m}$, respectively (shots 11,6 , and 8 ), and identify some of the main arrivals. The Scholte wave is the dispersed wave train arriving at later times. The general bandwidth for these waves was in the range of 0.3 to $6.0 \mathrm{~Hz}$.

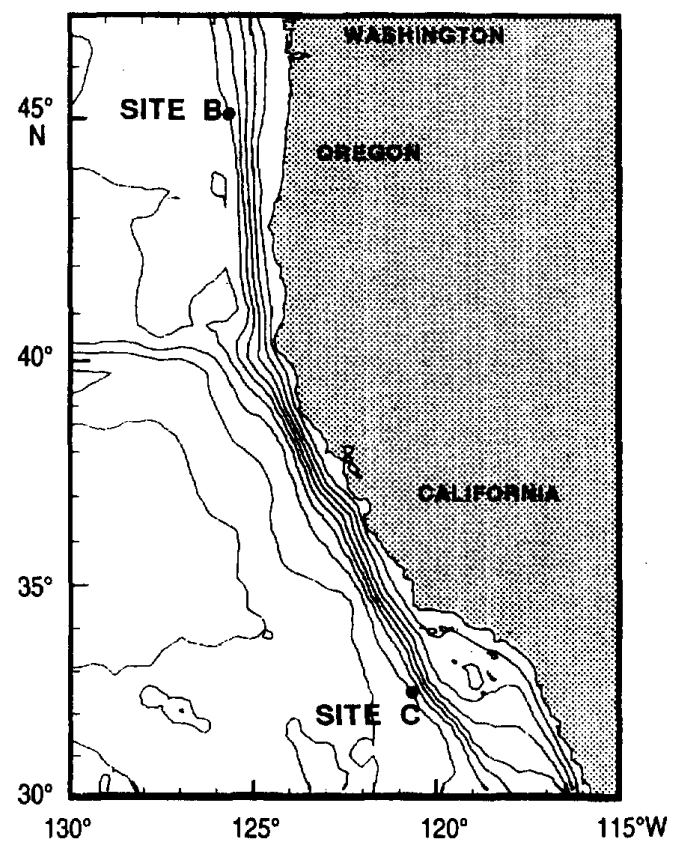

Fig. 1. Site locations for Scholte wave measurements used (after Bibee, 1993). 

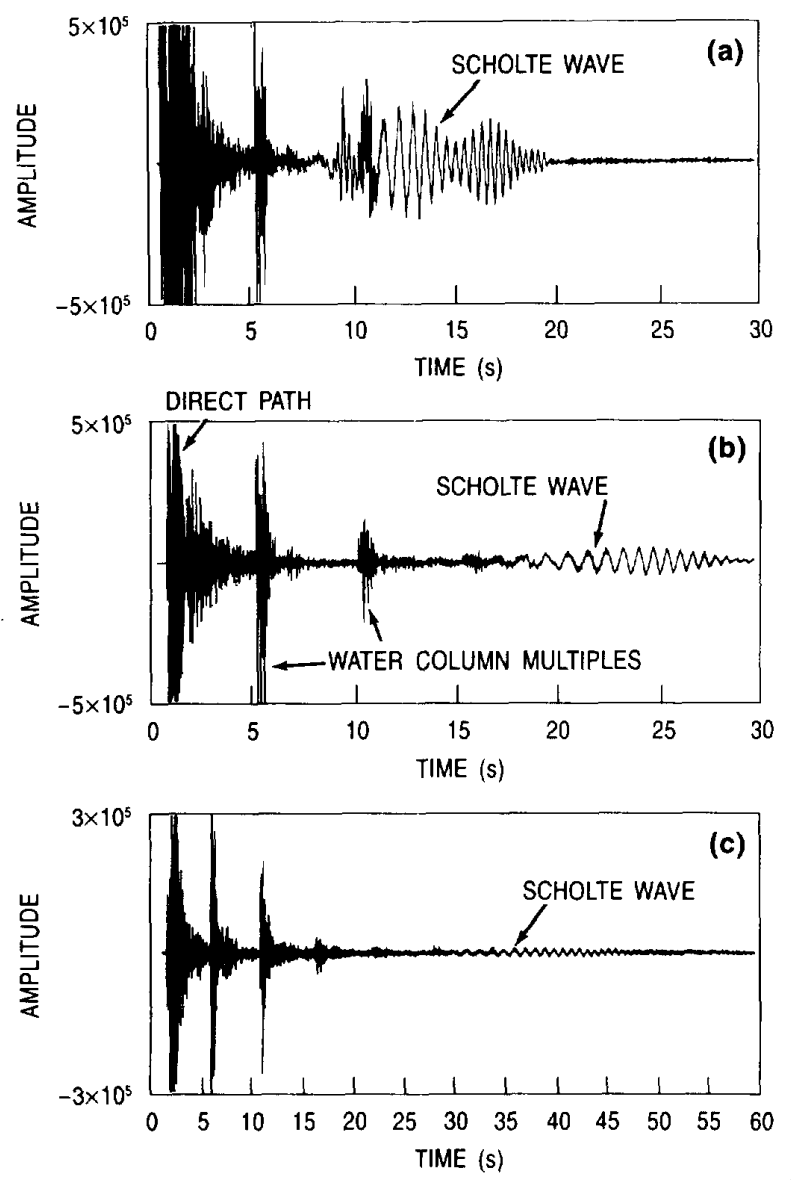

Fig. 2. Site C time series. All are from OBS 38 (a) shot 11, range $=620 \mathrm{~m}$, (b) shot 6 , range $=1190 \mathrm{~m}$ and (c) shot 8 , range $=2010 \mathrm{~m}$.

The low frequency energy content is governed by the attenuation properties of the sediments-i. e., the higher frequencies are rapidly attenuated.

In Fig. 3 (a) and (b), we display the time series recorded on OBS 34 for site B for ranges $990 \mathrm{~m}$ and $1910 \mathrm{~m}$, respectively (shots 3 and 1). The arrival times of the Scholte waves are smaller compared to those at site $\mathrm{C}$ as the sediment speeds are significantly greater at site $\mathrm{B}$. This is due to the terrigenous (sand) component of the sedimentation controlled by the Astoria fan.

\section{SPECTRAL RATIO METHOD}

Aki and Richards [11] define $Q$ in a way that leads to,

$$
A(t)=A_{0} e^{-\omega t / 2 Q_{t}} \text { or } A(x)=A_{0} e^{-k x / 2 Q_{s}},
$$

where $A$ is the observed wave amplitude at time $t$ or distance $x$, and spatial $Q_{s}$ and temporal $Q_{t}$ are related by
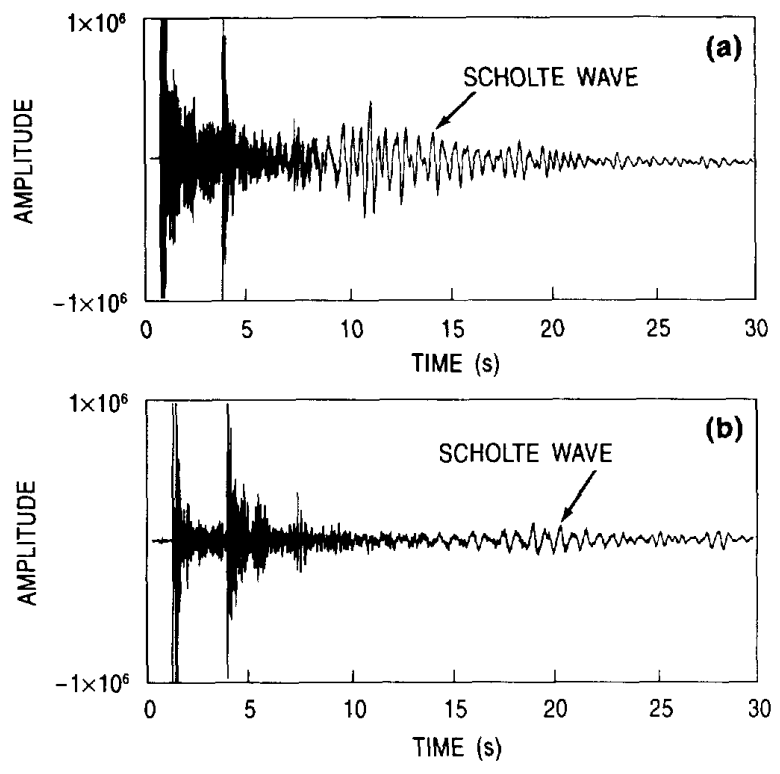

Fig. 3. Site B time series. Both are from OBS 34 (a) shot 3, range $=990 \mathrm{~m}$ and $(\mathrm{b})$ shot 1 , range $=1910 \mathrm{~m}$.

$Q_{t}=(c / U) Q_{s}[11],[12] . U(\omega)$ is the group velocity and $c(\omega)$ is the phase velocity. For non-dispersive propagation, there is no distinction between the two kinds of $Q$. We are estimating temporal $Q_{t}$ as we are using power spectra; however we are currently investigating use of the above relation to estimate $Q_{s}$ by computing $c(\omega)$ and $U(\omega)$ from modeling studies [5], [7].

Sauter, et al. [9], [10] define the power spectrum for the Scholte wave arrivals as,

$$
P_{s}(\omega)=|S(\omega) Y(\omega) R(r) G(\omega) I(\omega)|^{2}
$$

where $P_{s}$ is the Scholte wave power spectrum, $S$ is the source spectrum, $I$ is the instrument response, $Y$ is the vertical mode function which depends on the earth properties, $R$ is the geometrical spreading loss function, $r$ is range, and $G$ is the attenuation term,

$$
G(\omega)=e^{-\omega t / 2 Q_{t}} .
$$

If we isolate a particular mode, the spectral ratio of two Scholte wave arrivals $i$ and $j$ is then,

$$
P_{i}(\omega) / P_{j}(\omega)=\left(R_{i} / R_{j}\right)^{2} e^{\omega \Delta / / Q_{t}}
$$

where $\Delta t=t_{j}-t_{i}$. In canceling the $Y$ terms we have made the assumption of range independent earth properties. This is a reasonable assumption at site $\mathrm{C}$ due to the nature of the depositional processes. However, it may be a less adequate assumption at site $\mathrm{B}$. We also assume that the spectra are sufficiently similar from different shots that they cancel 
out. Justification for this assumption is made later. Finally, we should mention that the above procedure assumes isolation of a single mode. Our data is largely controlled by the fundamental mode, though no specific effort was made in this study to remove higher mode contributions.

Let the $\log$ spectral ratio $P_{l r}$ be defined as,

$$
P_{l r} \equiv \ln \left\{P_{i}(\omega) / P_{j}(\omega)\right\} \text {, }
$$

and define $\beta \equiv 2 \pi \Delta t / Q_{t}(\omega)$. If $\beta$ is a constant, then

$$
\frac{\partial P_{l r}}{\partial f}=\beta
$$

Therefore, $\beta$ is the slope of the $\log$ spectral ratio. For our data, $\beta$ is approximately a constant over the frequency range of principal spectral energy, so we can determine its numerical value from a linear least squares fit to $P_{l r}$ vs. frequency $f$.

\section{DESCRIPTION OF DATA ANALYSIS}

A rectangular window was applied to each time series analyzed (see Figs. 2 and 3 ) to separate the Scholte waves from other arrivals. Their respective autocorrelation functions were then computed. A smoothed power spectrum estimate was generated from each autocorrelation function in turn by multiplying it by an approximately 30 -second long Hamming window and computing the discrete Fourier transform. The presence of noise made the spectrum estimates unstable when computed directly by taking the modulus of the discrete Fourier transform of the Scholte waves. Slopes and their uncertainties, $\sigma_{\beta}$, were calculated from the $\log$ spectral ratio by standard least squares methods.

We estimated $\Delta t$ by taking the difference between the window centers of the ratio pairs. The center time of a particular Scholte wave packet was calculated by a weighted average over time, where the weights were constructed from a smoothed Hilbert envelope. This procedure also yielded variance estimates which we used in constructing the $\Delta t$ uncertainties $\sigma_{\Delta t}$. The final uncertainty estimate in $Q_{t}$ was determined from a standard formula in error analysis,

$$
\frac{\Delta Q_{t}}{Q_{t}}=\frac{\sigma_{\Delta t}}{\Delta t}+\frac{\sigma_{\beta}}{\beta}
$$

Table I provides the relevant quantities involved in the calculations.

The analysis we outline above needs one point of clarification. For dispersive propagation, the overall observed $Q_{t}$ in terms of the spectral ratio method is a function of frequency. This is apart from possible individual layer $Q$ frequency dependence, and does not imply such. This overall frequency dependence is simply due to the fact that interface waves have a penetration depth proportional to

\begin{tabular}{|c|c|c|c|c|c|c|}
\hline \multicolumn{7}{|c|}{ Site C } \\
\hline Ratio & $\Delta r(m)$ & $\Delta t(\mathrm{~s})$ & $\beta(s)$ & $Q$ & $\begin{array}{c}\text { Relative } \\
\text { Error }\end{array}$ & $x(\mathrm{~dB} / \lambda)$ \\
\hline$\$ 11 / \$ 8$ & 1390 & $24 \pm 3$ & $4.8 \pm 0.1$ & $31 \pm 5$ & $16 \%$ & $0.88 \pm 0.14$ \\
\hline S6/S8 & 820 & $14 \pm 3$ & $3.0 \pm 0.1$ & $29 \pm 7$ & $24 \%$ & $0.94 \pm 0.23$ \\
\hline S11/S6 & 570 & $10 \pm 2$ & $1.6 \pm 0.1$ & $39 \pm 10$ & $26 \%$ & $0.70 \pm 0.18$ \\
\hline \multicolumn{7}{|c|}{ Site B } \\
\hline S3/S1 & 920 & $10_{ \pm 4}$ & $2.2 \pm 0.2$ & $29 \pm 14$ & $48 \%$ & $0.94 \pm 0.45$ \\
\hline
\end{tabular}

TABLE I

RESULTS FOR SITE C AND B DATA ANALYSIS

wavelength, and for dispersive propagation (caused by increasing shear speeds with depth), different wavelengths will "see" different $Q(z)$ profiles. The Scholte waves in our measurements are dispersive, and the method we outline above for computing $\Delta t$ amounts to a weighted average of $Q(\omega)$ over frequency. This was verified through a dispersion analysis of the data.

\section{DISCUSSION OF RESULTS}

In Fig. 4 (a)-(c), we display the log spectral ratios and their least squares linear fits vs. frequency for the site $\mathrm{C}$ data. The log spectral ratios are for shot 11 and shot 8 , shot 6 and shot 8 , and shot 11 and shot 6 , respectively. In Fig. 5, we show the log spectral ratio for site B, where we used the ratio of shot 3 to shot 1 . The spectral ratio variance is noticeably higher for the site B case. This is possibly due to a violation of the assumptions we made for the spectral ratio method. In Table I we list the $Q_{t}$ estimates using these three cases. The range separations for site $\mathrm{C}$ are between $570 \mathrm{~m}$ and $1390 \mathrm{~m}$. The most accurate $Q_{t}$ estimate was for the shot 11 to shot 8 ratio, and had a value of $31 \pm 5$. The relative error was $16 \%$. The other two ratios produced estimates of $29 \pm 7$ with a relative error of $24 \%$, and $39 \pm 10$ with a relative error of $26 \%$. At site B the sediment shear speeds were roughly twice those at site $C$ [5], [7] so twice the range separation would be needed to get the same absolute wave attenuation as site $C$ if the mean attenuations for the two sites were about the same. Our maximum range separation at site B was $920 \mathrm{~m}$, which was just adequate to get one usable result. Fig. 5 displays the spectral ratio for shots 3 and 1 , and the $Q_{t}$ estimate for site B is $29 \pm 14$ with a relative error of $48 \%$ (refer to Table I). The low accuracy of this result prevents us from comparing the results for sites B and $\mathrm{C}$.

We remark that the final uncertainty computed for each $Q_{t}$ estimate was largely governed by $\sigma_{\Delta t}$. This later quantity was difficult to estimate in an objective and statistically sound manner. This was partially due to the lack of redundancy in the experiment, and mainly due to the dispersive 

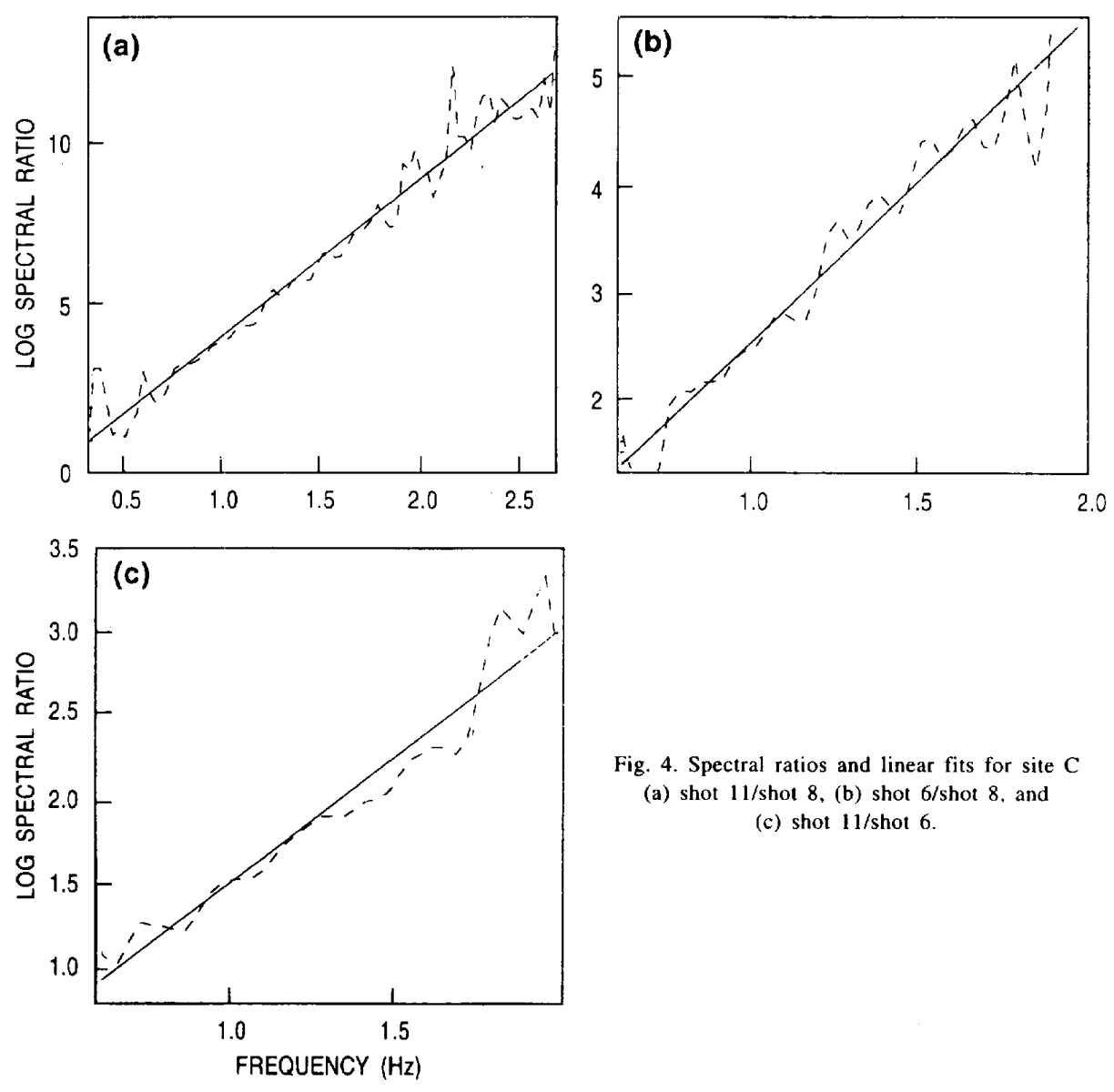

Fig. 4. Spectral ratios and linear fits for site $\mathrm{C}$

(a) shot $11 /$ shot 8 , (b) shot $6 /$ shot 8 , and

(c) shot $11 /$ shot 6 .

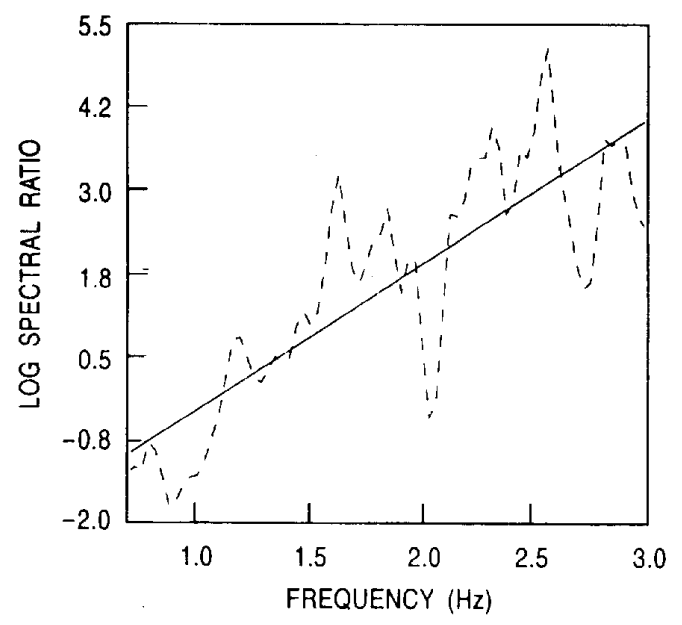

efforts. It should also be possible to considerably refine the $Q_{t}$ estimates through numerical inversion procedures [5], [7].

Jensen and Schmidt [3] collected various estimates of shear attenuation $\gamma$ from the literature. They report a general range for Scholte wave measurements of $0.02-2.3 \mathrm{~dB} / \lambda$ and $0.5-2.0 \mathrm{~dB} / \lambda$ for in situ measurements, where $Q$ is related to attenuation $\gamma$ by $\gamma=27.29 / Q$. Conversion of our results gives a general range of $0.49-1.39 \mathrm{~dB} / \lambda$, which is well within the range of literature values (see Table II). A more site-specific comparison can be made for site $\mathrm{C}$, as Sauter et al. [9], [10] report results for that area. Their estimates are reported in Table II, along with our best

TABLE II

RESULTS FROM SAUTER ET AL. DATA ANALYSIS

Fig. 5. Spectral ratio and linear fit for site B, shot 3/shot 1 .

nature of the Scholte waves. The estimation of $\Delta t$ and its uncertainty could possibly be addressed through modeling 
determination of their range separations. The weighted mean of our results for site $\mathrm{C}$ is $Q_{t}=32$, and we calculate their weighted mean to be $Q_{t}=18$. Our average $Q_{t}$ estimates are larger by a factor of 1.8. Sauter et al.'s [9], [10] results from the ratios do not all agree internally (overlap). Possible explanations, but no definitive conclusions, are provided for the apparent inconsistencies in their results. One of their results agrees well with our lowest error result. We should point out that our range separations were greater than theirs (compare Table I and II) on average by a factor of -2 . Thus, our measurements are biased towards lower frequencies relative to theirs. This causes our Scholte waves to have greater penetration depths. It is generally believed [3] that shear $Q$ increases with depth, which would partially explain our higher $Q_{t}$ values. It should also be pointed out that these were two different test sites, albeit within the same area. Also, we have made an assumption about the shot spectra being similar so that their effects cancel in the spectral ratio. Sauter et al. were able to produce ratios from the same shot. Nevertheless, the linear nature of our spectral ratios compares well with theirs, giving some justification of our assumption of shot repeatability.

\section{CONCLUSIONS}

We have analyzed Scholte wave measurements from two diverse test sites to gain estimates of shear $Q_{r}$. The quantities obtained are averages at two levels. $Q(\omega)$ is related to the individual layer $Q(z)$ 's by a complicated, weighted average over depth. This averaging is done by the earth. We then calculate $Q_{t}$ in a way that amounts to averaging $Q(\omega)$. The $Q_{2}$ estimates fall within the range of $29-49$ for site $\mathrm{C}$ and 15-43 for site $B$. The analysis makes two general assumptions: (1) the sediments are laterally homogeneous, and (2) the shot spectra were repeatable. The governing factor in the final uncertainty estimates for the calculated $Q_{t}$ values was $\sigma_{\Delta t}$.

\section{ACKNOWLEDGMENTS}

This work was supported by the Naval Research Laboratory (6.1) basic research program, Program Element $0601153 \mathrm{~N}$, Subelement 32. NRL contribution number NRL/PP/7173--93-0019.

\section{REFERENCES}

[1] B. Schmalfeld and D. Rauch, "Explosion-Generated Seismic Interface Waves in Shallow Water: Experimental Results," Report SR-71, SACLANT ASW Research Centre, La Spezia, Italy, 1983.

[2] H. Ali and B. Schmalfeldt, "Seismic Sensing of Low-Frequency Radiated Ship Noise," Report SR-77, SACLANT ASW Research Centre, La Spezia, Italy, 1984.

[3] F. B. Jensen and H. Schmidt, "Shear properties of ocean sediments determined from numerical modelling of Scholte wave data," in Ocean Seismo-Acoustics, T. Akal and J. M. Berkson, Eds., New York: Plenum Press, 1986, pp. 683-692.

[4] M. Snoek, "Interface-Wave Propagation Studies: An Example of Seismo-Acoustic Propagation in Non-Homogeneous Materials," Report SM-229, SACLANT ASW Research Centre, La Spezia, Italy, 1990.

[5] H. B. Ali and L. D. Bibee, "The influence of sediment layering and geoacoustics on the propagation of Scholte interface waves," Proc. OCEANS 93, IEEE.

[6] H. B. Ali, L. D. Bibee, and J. L. Becklehimer, "Observations of Scholte wave dispersion from measurements at two diverse test sites," Proc. OCEANS 92, IEEE, 1992.

[7] L. D. Bibee, "In situ measurements of seafloor shear-wave velocity and attenuation using seismic interface waves," in Acoustic Classification and Mapping of the Seabed, Proc. of the Institute of Acoustics, Bath UK, Vol. 15, Pt 2, pp. 33-40, 1993.

[8] R. S. Jacobson, G. G. Shor, and L. M. Dorman, "Linear inversion of body wave data - Part II: Attenuation versus depth using spectral ratios," Geophysics, vol. 46, No. 2, pp. 152-162, 1981.

[9] A. W. Sauter, L. M. Dorman, and A. E. Schreiner, "A study of sea floor structure using ocean bottom shots and receivers," in Ocean Seismo-Acoustics, T. Akal and J. M. Berkson, Eds., New York: Plenum Press, 1986.

[10] A. W. Sauter, "Studies of the Upper Oceanic Floor using Ocean Bottom Seismometers," Dissertation, U. Calif., San Diego, 1987.

[11] K. Aki and P. G. Richards, Quantitative Seismology: Theory and Methods, vol. I, Freeman, San Francisco, 1980.

[12] J. N. Brune, "Attenuation of dispersed wave trains," Bul. Seism. Soc. Am., vol. 52, No. 1, pp. 109-112, January 1962. 